\author{
Thing Theory: Connecting Humans to Smart Healthcare \\ Sally A. Applin (University of Kent - Canterbury, UK) and Michael D. Fischer \\ (University of Kent - Canterbury, UK) \\ Source Title: Internet of Things and Advanced Application in Healthcare \\ Copyright: (C) 2017 Pages: 17 \\ DOI: 10.4018/978-1-5225-1820-4.ch009
}

\title{
INTRODUCTION
}

Healthcare providers will enter location-aware smart environments with the expectation that their devices will integrate, their location will be incorporated, and the environment that they are within will specifically respond to their needs, as well as to the needs of their patients. Cooperation and coordination in complex environments requires people to have access to appropriate contextually sensitive information, some of which must be shared between them. To plan and design effective location-aware smart environments for healthcare, tools are required for integrating and responding to human needs and anticipating human intents and desires.

Humans depend upon successful cooperation with each other for their survival. As humans have evolved their environments, their communications structure and the messages that it produces have become more complex. Sharing or overlap of messages becomes critical in these highly heterogeneous environments, now comprised of people with many points-of-view using a range of channels for communication in multiple languages.

A location-aware healthcare smart environment is another layer within this already highly heterogeneous system of communication. Each component in a location-aware smart environment network can generate data and send messages that must be processed, understood and responded to in some manner. In a healthcare environment, well placed software agents can help manage critical messages shared between sensors, low level software agents and the people who act on this information, improving care for patients and outcomes for providers.

The authors' propose a framework based on the agency of both humans and environmental components: Thing Theory, a logic-based agent framework that evolves discussion on how to connect humans to a healthcare environment designed to function for their benefit.

\section{BACKGROUND: THE HEALTHCARE ENVIRONMENT}

Discrete pieces of the healthcare environment can be automated with Internet of Things (IoT) technologies, lessening the risk of mistakes from the outcome of multiple, multiplexed messaging and increasing the cooperation and abilities of technology to partner with people. In ideation, smart environments in healthcare are a great idea. In practice, much must be overcome for their successful deployment (Perry et. al 2013). Healthcare is a legacy system, largely regulated by government. In healthcare, many mistakes can have serious long term consequences, or can be fatal. Thus, any automated element within the healthcare domain must be well thought-out, robust and offer redundancy to enable successful application (Fairbanks et. al. 2014).

With the IoT and healthcare there are many possible hypothetical scenarios that could be developed, from the critical, such as smart operating rooms, pharmacy services, and smart rooms for patient or psychiatric care units (Wears \& Leveson 2008), to more mundane aspects of healthcare such as sections of preventative clinics or cafeteria and parking services. In healthcare, using the IoT for patient care and using the IoT to reduce costs can co-exist as mutual goals to improve healthcare quality, as joint benefits emerge from streamlining for efficiency and improvement of service quality (Chaudhry et. al. 2006). IoT strategies for healthcare should enhance and leverage legacy systems rather than reduce services as a by-product of automation.

The easiest early applications of the IoT could augment current automated elements of medical processes. An IoT within healthcare should be based on two broad concepts, distribution of functions to addressable devices where each device is responsible for a limited set of operations or capabilities, and a framework for flexibly integrating these devices so that developers, or even end users, can assemble the set of capabilities needed for some situational macro-task. Thus, IoT devices are tools that can be used for a vast range of purposes, rather than integrated systems per se. Integration 
emerges from instantiation of a given tool assemblage into a process through knowledge-based systems external to each device (Bradshaw et. al. 2013). This orientation largely mirrors the current situation, where space and individual tools are available for highly trained teams of individuals to integrate as needed to serve different medical procedures.

Minimally, connecting a device to the IoT framework requires transforming the external information a device produces and consumes into a form that can be transmitted over a network (Gubbi et. al. 2013). Examples of relatively straightforward healthcare IoT applications enable scales, blood pressure monitors, temperature and other visit quantification devices to share data directly by transmitting on demand usable measurements to a requesting (or watching) network agent. One or more network agents could manage the patient's record from each device. For example, as a patient enters a room, the room could be either activated by sensor, or could activate when a healthcare worker logs into the room's network and verifies the patient identity in the room. As the healthcare worker takes the measurements on various devices, the smart machines can send their readings to an open file, with buttons on the devices as options to skip logging the reading, or a way to do that in software in case there is a patient request to not update certain readings. Automating this data entry would save the time of the healthcare worker, who currently must scribe and re-enter the data into the computer after completing the data collection with the patient.

Most current devices used in medical contexts are designed with the intent to be administered by highly trained people. Current devices need to be actuated to take a useful measurement and to have the appropriate context for that measurement to be useful. Some of these tasks are easier to address than others. An example of this might be to use IoT sensors to maintain room supply inventories. A simple sensor could determine if a supply level was low for cotton or alcohol pads, or other supplies, and this could trigger inventory resupply. In small clinics, this may not be useful, but in larger hospital settings, it may be helpful to have supply levels monitored and automated for refills. IoT sensors can monitor if someone is out of their bed, if the door has been opened, if the floor has extra weight on it, if the lavatory has been used or not, etc.

What can be measured, recorded and sent back as data in the healthcare environment is only limited by human imagination. What will be key to IoT healthcare scenarios are trusted methods to manage this low-level sensor data as messages between agents (Babar et. al. 2010). In addition, the data from these messages would serve the patient and clinics best if it could be managed and integrated in more meaningful ways that contribute to the well being of the patient and the efficiency of the healthcare enterprise. In healthcare especially, context of data collection is critical (Ahsan et. al. 2009).

If there is a patient who is mobile, and staying overnight in a room for observations or tests, whether or not they get up to use the lavatory (assuming it is not related to their tests) is less important in a smart environment, than if a patient records using the lavatory every 15 minutes and does not have a condition precluding that behavior. Simultaneously, patient data must be protected against uses that do not serve patient treatment, and in cases where it does, care must be taken so that the patient does not feel that they are a part of a larger surveillance state, only benefitting the healthcare clinic or provider, rather than patient well-being.

To achieve this in an IoT multi-agent healthcare environment, a trusted broker between individual agents and components and the healthcare workers and patients, is required to achieve secure and appropriate results. Thus, to move healthcare into the IoT realm, a universal 'thing' that can function as a source of trust and competence to organize other agents (Atzori et. al. 2011) while securing and protecting patient data, is required.

\section{THING}

\section{An Agent Based Multiplexed Message Manager}

The 1960's Addams Family television series was based on a cartoon by Charles Addams (The Paley Center for Media, n.d.). The Addams Family lived in an enormous old mansion, full of taxidermy and other curiosities, located adjacent to a cemetery near caves, quicksand, and a swamp. The character of Thing is a disembodied hand (and forearm) that has been with the family for many years and is described as both a 'family retainer' and 'friend.' It inhabits a series of tabletop boxes in different rooms of the house that could be compared to a type of roughly cobbled physical network. 
Thing also inhabits plant pots, clocks, the breadbox, glove compartments, tree roots, and other devices to use as a base for interaction. Thing communicates with the family by gestures, sign language, writing out notes, or tapping out messages in Morse code. Thing serves the family by accessing a portal in contextual proximity to what is needed. Thing will answer the phone, by taking the receiver off the hook (and later replacing it), pour the tea, retrieve and deliver the mail, play castanet accompaniment to the Butler's harpsichord recitals, light cigars, return hats, offer advice, put its finger on a bow for tying up a present, or whatever else might be needed or desired at the precise moment required, in the precise room or context needed. Thing is not only a ubiquitous agent, but an anticipatory one that migrates within the environment. Although the family displays a "Beware of the Thing" sign on their front gate, Thing is shown to be courteous, friendly and helpful.

The authors consider the Addams Family's Thing, minus the Uncanny Valley issues (Masahiro, 1970), as a good potential starting point for how agent behavior and interaction could support people in location-aware smart environments, including those within healthcare. The sensing, response and location-awareness of Thing is a useful aspirational model, even if the goal is not for disembodied hands to pop out of boxes to assist a patient. As an agent of sorts, Thing learned about and possessed data knowledge of every member of the household, their preferences, needs and desires, and was sensing and aware as to what type of contribution it could make to the successful outcome of their actions. Thing worked with their location and required nothing from them to be able to assist them, outside of their accepting its non-verbal limitation.

Thing had sensors, data, and was location-aware. It also managed in part, the relationships between members of the household. This is what the authors would expect of an agent in a healthcare smart environment, and what the authors hope, within the range of sensitivity to privacy, might be achieved. Developers will have to think like Thing in order to take into account how to manage the multiplexed communications, needs and messages that come from multiple sensors and actuators at different locations within the healthcare environment. People, sensors or actuators might be moving within the environment requiring location awareness on the part of the overall environment to use the capabilities of the space. Physical spaces are gradually shifting, with the aid of pervasive technology, from being mainly locations or destinations (with space in-between), towards the notion of being places that 'host transitions' (Applin and Fischer, 2011a).

In healthcare, this becomes critical as people may move through many spaces as a result of what is required for their care. A patient might begin in one environment (doctor's office) and transition through the course of diagnosis or treatment through several more offices, labs, or specialty clinics, each for an unknown amount of time. No two patients may have the exact time or place experience as part of the process of their care. This makes provider-to-patient ratios difficult to estimate and creates an unpredictable system that when unified for a 'standard' patient, risks individualized care through poor design of over homogenization. However, smart environments that can keep track of individual needs, requirements, times, preferences and schedules, can be welcome additions to any healthcare system, if they are implemented in a way that promotes human agency and protects patient privacy. In other words, it becomes even more critical for developers to create agency-based aware systems and to design the algorithmic underpinnings of the healthcare smart environment to be useful, yet unobtrusive. Most critically, these new designs must integrate to work within existing legacy systems.

\section{PERVASIVENESS AND POLYSOCIAL REALITY}

When the authors examined the current state of the distribution of pervasive technology, they discovered that it permeates some areas, and barely covers others. As services by sensors and actuators becomes pervasive, human expectation, and some level of fusion with the systems that support sensors, will become more and more intertwined.

To smooth the transition for healthcare into a sensor/actuator services system, conflicts within its culture with regard to adopting and adapting new technologies must be resolved by those developing and deploying pervasive technologies. Technological practice that is 'marked' (e.g. unabsorbed) cannot be pervasive. Technologies becoming 'unmarked' (e.g. absorbed) into the 'unawareness' of daily life in a healthcare system, indicate successful technology acceptance (Applin and Fischer, 2011a). 
Humans are constantly trying to communicate whether static or in motion or in proximity or distanced. When humans communicate with others they might talk through closed doors. They also often disrupt, interrupt and try their best to be heard and to listen. Digital mobile technology enables people to be in the same place at the same time, or not, and to be mobile or not. Due to time, space, and conceptual differences, people may not be in the same 'plane of reception' at the same time even if they share time and space coordinates. To the extent that people share common sources of information while interacting with each other, the greater their capacity to collaborate becomes. If humans share too few channels relevant to a common goal, there may be too little mutual information about a transaction to interact and communicate well collaboratively. Poor collaborative interaction can lead to further relational fragmentation with the potential to promote isolated individuation on a broad scale (Applin and Fischer, 2011b). By changing the means that humans use to manage space and time during their daily routines, developers can shift human experience from individuated, user experiences to enhanced sociability within a multi-user, multiple application, multiplexed messaging environment. In healthcare smart environments, there are multiple channels creating multiple communications, which may or may not be coordinated or multiplexed, and receiving multiple communications in kind that may or may not be synchronous, all while people may be moving through the environment. It can add up quickly to being overwhelming (Applin and Fischer, 2012a).

PolySocial Reality (PoSR) as a conceptual model of the global interaction context that emerges when use of the social mobile web and other forms of communication contribute significantly to instantiating intentions (Applin and Fischer, 2011b, 2012a). PoSR describes the aggregate of all the experienced 'locations' and 'communications' of all individual people in multiple networks and/or locales at the same or different times. PoSR is based upon the core concept that dynamic relational structures emerge from the aggregate of multiplexed asynchronous or synchronous data creations of all individuals within the domain of networked, non-networked, and/or local experiences (Applin and Fischer, 2012b).

Network and mobile communications tend towards message multiplexing that results in a PoSR messaging environment that goes well beyond the limitations of human physiological systems to directly engage (Applin and Fischer, 2012b). Thus, the interaction environment described by PoSR implies that there are great challenges in using upcoming technologies to improve the social integration of people and their environments, and the entropy associated with location-awareness combined with PoSR outcomes creates a complexity problem that might benefit from a particular kind of Artificial Intelligence (AI) agency to solve on an as needed basis. When environments have the potential to be 'social' (even between themselves as machine-to-machine) there exists a high potential for fragmentation (e.g. partial or complete isolation from ensuing social transactions) due to PoSR related multiplexing.

This type of messaging situation in healthcare can be critical and lead to fatalities if messages communicating urgent, specific care instructions are not received and acted upon in a timely manner. The capacity to parse multiple messages in location-aware healthcare environments is certainly possible, through sensors and processors, and collating that material and sifting through what is critical is where an integrative agent can contribute. This is where Thing excels as an agent, mostly based on knowledge of the situation, the requirements and specifications, and what is going on in context in real time; the capabilities of the healthcare environment in the present. There will need to be well considered somewhat transparent software and hardware in order to design agents with the utility of 'Thing' to manage multiplexed communications, needs and relationships in the healthcare environment.

\section{THING THEORY}

Thing operates through observing, reasoning and then taking action. Thing's observations and reasoning are based on knowledge of the humans in its proximity that it has gained over time through understanding and learning what type of tasks they require assistance with on a regular basis. Thing expands the agency of group members and greatly enhances their experience within their rather complex environment by acting as an agent on behalf of a wide range of different services and facilities the environment has to offer, connecting these to intentions of the group. In a healthcare environment, Thing would require trusted access (on an appropriate sliding scale depending upon patient healthcare needs) to learn about a patient in order to successfully assist it in the healthcare 
environment. Thing can transform a complex jumble of services into a successful technological context. The authors argue that successful technological contexts are those that people use to expand their agency outside that technological context proper; the technology expands their general capacity to choose. (Applin and Fischer 2013).

A Thing-agent might improve a healthcare smart environment by specifying some principles that Thing must satisfy. Foremost, the authors would argue that a Thing-agent can and will facilitate people exercising agency.

Agency is the capacity to make and execute nondeterministic choices intended to advance to a goal as events unfold. For example, humans exercise agency when deciding whether to turn on a light or to walk across a street to avoid a possible obstacle. Agency implies that agents' future choices are not intrinsically fixed or stochastically predictable except on the basis of secondary principles of reasoning, such as rationality, cooperation or enmity.

In any given circumstance, people have a set of options upon which they could base choices. In many cases they will not be aware of some options, a situation that discovery might advance. In other cases, people will lack the means to usefully enact an option because of a lack of skill or knowledge, even though they are aware of it as an available possibility. In still other cases, options might not be available because the contexts within which these would be available, cannot be deployed. Agency is a direct function of how well people can exercise available options into choices. Agency can be expanded by making new options available by discovery or invention, or through supplying the knowledge and skill required to exercise an option. Agency can be reduced by removing options or the capacity to exercise options. We can measure the effective agency within a context relative to a given agent as a simple ratio, choices/options.

Often these options and corresponding choices are somewhat predictable due to the assumption of cultural and social frames for reasoning that will vary between people depending on social roles, relative power and specialist knowledge. High levels of predictability will be based on mutual understanding of the different points-of-view by each participant. Resolving most complex situations is not as simple as making a single choice or a simple sequence of choices.

Adaptive agency is the capacity to transform options into choices, sometimes through learning or skill, but most often through exchange with other agents, where each possesses knowledge and skills that can activate options on behalf of the other. Exchange can be direct or deferred and results in the establishment of social relationships between agents. The foundation of a social relationship requires a mutual presumption of agency on the part of the other; social relations require that each party assumes the other has some level of agency (Applin and Fischer, 2012b). One of the outcomes of this relationship is a process of negotiation between social agents to arrive at a mutually beneficial consensus that influences future choices.

If someone wanted to turn on a lamp, they would most likely try a wall switch, the switch on the lamp, check the plug, or in rare cases, depending upon TV exposure, clap their hands (Srinivas et al., 2004). An average person might not expect or anticipate that the person seeking illumination would put their head in the refrigerator to make use of its automatic light. However, humans can, and have made this choice at times. This is the difficulty in developing adaptive software for an Agent (Thing). Fixed decision tree choices are good, but do not have enough information within them to fully satisfy human agency. An environment, to be truly smart, must learn from the cumulative data within its realm to understand and guess what likely choices might be for a given agent and then facilitate or enact these on behalf of that agent.

The first principle of Thing Theory shown in Table 1 is that the Thing-agent operates as a meta-agent over the entire technology context, not as a sub-component. Our Thing-agent assembles capabilities (e.g. whether or not the refrigerator light is suitable as a lamp) that are extensible based on what subcomponents of the system happen to be available. What Thing can do is ultimately limited by the basic capabilities of various system subcomponents in combination with Thing's knowledge about these and how to combine capabilities to make new more context sensitive capabilities. To increase the Thing-agent's capabilities, more information from subcomponents must be shared. The third principle of Thing Theory is that the Thing-agent must be context aware and able to identify that different combinations of capabilities are available in different contexts, and have a corresponding capacity to manipulate contexts (e.g. enact, repress, aggregate) to 'reveal' new capabilities, many of 
which may be 'innovations' based on context discovery (invention). The fourth principle of Thing Theory is that a Thing-agent extends the capabilities of other meta-agents. In order for the fourth principle to work, the meta-agents (a social network of at least one Thing-agent and another metagent) must have some type of transparency or at least shared permissions for the exchange of capabilities and contexts. To describe or analyze such multi-agent systems in healthcare, developers must take account of the social and well as the individual behaviors of the agents as well (Dix and Fischer, 2011).

In implementing Thing theory in location-aware Smart Environments the authors argue that a Thingagent must relate to other agents an interface between multiple smart technological contexts. At a minimum, the Thing-agent will inform agents of the capabilities of a Smart Environment in pragmatic terms that make sense to the agents, and ideally provides a high level interface for the agents into these environments. (Applin and Fischer 2013).

The Thing-agent will discover the capabilities of the various systems it ranges over, and possess a representation of the pragmatic contexts for these. There is an inherent notion of extensibility; new features will be added and people (or other agents) will use the these for many purposes not anticipated by designers. A Thing-agent could more forward on this problem by using an extensible multi-agent simulation incorporating a specification of the capabilities of each sensor and actuator, user expertise and other information, to create a model of the outcomes of how services and agents might interact with each other. Thing-agent could then offer reasonable choices to user agents in terms of what is likely to occur should the agent make a given choice. (Applin and Fischer 2013: Conclusion).

Table 1. Principles and Functions of the Thing-agent

\begin{tabular}{|c|c|c|}
\hline & Principle & Function \\
\hline 1 & Thing-agent is meta agent & Operates over entire context \\
\hline 2 & $\begin{array}{l}\text { Thing-agents's capabilities are } \\
\text { based on system } \\
\text { subcomponents. }\end{array}$ & $\begin{array}{l}\text { Thing-agent's capabilities of } \\
\text { extension are limited by } \\
\text { system subcomponents } \\
\text { capabilities. }\end{array}$ \\
\hline 3 & $\begin{array}{l}\text { Thing-agent must be context } \\
\text { aware. }\end{array}$ & $\begin{array}{l}\text { Thing-agent must be able to } \\
\text { identify different capabilities } \\
\text { in different contexts and to } \\
\text { select most appropriate one to } \\
\text { take action. }\end{array}$ \\
\hline 4 & $\begin{array}{l}\text { Thing-agent works with other } \\
\text { meta agents }\end{array}$ & $\begin{array}{l}\text { Thing-agent works with other } \\
\text { meta-agents to expand their } \\
\text { capabilities when required. } \\
\text { Shared permissions/context/ } \\
\text { capabilities are required as is } \\
\text { some transparency between } \\
\text { other meta-agents. }\end{array}$ \\
\hline
\end{tabular}

\section{IMPLEMENTING THING THEORY}

There are two approaches to implementing Thing theory in healthcare smart environments: as an interface to user-agents; and as an interface between multiple smart technological contexts that may be using and supporting the same location. A Thing-agent must do both to be effective, where the Thing-agent serves at a minimum as a means to inform user-agents of the capabilities of the smart environment in pragmatic terms that make sense to the user-agents, and ideally provides a high level interface to these. The Thing-agent must be able to discover the capabilities of the various sub- 
systems it ranges over, and to possess a representation of the pragmatic contexts in which the capabilities are expressed. This must be done in a way to where Thing-agent facilitates choices by user-agents, rather than forcing user-agents into specific choices.

For most technology, the user-agent interface is designed by implementing a metaphorical interface. This works reasonably well for simple technological contexts, though it often takes a very long time for designers to find just the 'right' metaphor for implementation.

In a healthcare smart environment, there is usually the intent to 'embed' a technological context within existing contexts that people use. Thus, there is an inherent notion of extensibility in that new features can be added, sometimes arbitrarily with the assumption that people will use the environment for many purposes, not all of which can be anticipated by designers. A possible approach for resolving this is for Thing-agent to employ one or more extensible multi-agent simulations that incorporate a specification of each sub-system that specifies sensor information, associated services and the role of other agents, including health care professionals and patients, to project multiple hypothetical models of how the services and agents might interact with each other, and the resulting contexts that emerge from different combinations of interaction. This would provide a basis for Thing-agent to offer informed choices to both sub-system agents and user-agents, providing feedback in advance to them regarding the choices and the likely outcomes should an agent make a choice, thus permitting agents to 'fine-tune' how they proceed.

To achieve this, developers might need to move beyond classical imperative logics most commonly in use for inference-based systems. Deontic logic was originally developed to explore the logic of morality in theology. In addition to the classical predicate calculus, deontic logic adds two basic modal operators, permitted and obliged (and their negations). Rather than describing specific outcomes, deontic operators focus on potentialities such as what is permitted and not permitted at a given time, with the capacity to identify derivations that make the possible impossible, and the impossible possible. From an agency perspective, identifying available options and choices, and in particular a means of analyzing what is needed to turn a theoretical option into a practical choice (Fischer, 2006).

Deontic logic has been demonstrated to be a useful basis for constructing simulations of sensitive realtime, time and location aware interactions between agents of different types (Ryan et. al. 1991; Dong and Li 2013). Casto and Maibaum (2007) present a deontic logic suitable for representing the interrelations between agents of different types, including agents exhibiting agency, and Horty (2001) is an extended logical treatment of representing and reasoning with agency in deontic logic. An advantage of modeling with deontic logic is that it is relatively easy to introduce new agents, new conditions and new outcomes into a working simulation and produce results that are instructive to user-agents.

Furthermore, a Thing-agent mediated multi-agent simulation can be useful for designing new pancontext technologies to be incorporated into the location-aware smart environment, since these too can take advantage of 'simulation services' provided by Thing-agent as a part of their decision making, both discovering new capabilities, contexts and a basis for comparing different options. Effectively this facilitates the incorporation of true agency into even sub-systems, and thus the capacity for the design of social cooperation between processes, rather than master-slave relationships, exploiting Thing-agent to ensure that the information that needs to be shared to support agency in the network of agents is available to maximize the capacity for cooperation, and avoid the problems that can arise from PoSR networks of cooperating agents.

A presumption might be made that a Thing-agent must have a human intelligence of sorts. The authors agree to the extent that social intelligence is human. However, research such as Brian Hare's ongoing research on dogs highlights their non-human anticipatory intelligence as a alternate type of sensory intelligence (Hare and Tomasello, 2005). A Thing-agent needs to resolve design principles and may need to do this in non-human ways, while remaining socially aware.

\section{Patient Process Management Using Thing Theory}

Extending the Kaiser and IBM example from the IBM Interconnect Smarter Processes Keynote in 2015 (IBM Software, 2015), one of many possible cases where Thing Theory could be used is as a solution to nurse-to-patient room ratio concerns. In the Interconnect example, nurse to patient ratios 
are shown to need to be maintained at 1:4 for optimum care in a hospital environment. The IBM/ Kaiser solution proposed tracking nurses to trigger messaging systems, to direct available staff to rooms on wards with lower ratios. This tracking may cause privacy concerns for nursing staff and inhibit implementation. If the healthcare provider's concern is insuring that patient needs are attended to, Thing-agents could monitor in-room processes at a sensor/process level and communicate those to negotiate with other Thing-agent representatives to summon staff.

One critical element of patient care is that the processes required to maintain that specific conditions of care, possibly unique to a single patient, are fulfilled. In the case of hospitalization, each patient may have different needs. Some may need oxygen, heart rate, fluid monitoring at close intervals, others may need different systems monitored or the same ones at different intervals. As people and their conditions are individualized, so must be their care. This is challenging to manage in an environment that must operate within constrained budgets and resources.

A new way to relate to patients using the IoT could be to understand patient care needs as time intervals and time functions that are different from each other. Computing gives developers tools to enable more sophisticated time management. A Thing-agent can manage the time schedules for each patient's needs and communicate those with other Thing-agents that manage nurse schedules. In this way, hospitals would not be tracking whether or not nurses were on the floor, but rather whether or not the maintenance tasks for individual care were addressed at the times they needed to be addressed. The sensors themselves on devices would respond to checks in place by physically present nurses, who would be available to check these patients due to the needs of the monitoring, rather than only due to needs of a nurse to patient ratio.

\section{CONCLUSION}

The healthcare environment intersects with people in deep and meaningful ways. To automate it without this consideration can be dangerous. The first steps of automation as applied to healthcare environments have been based on 'lean' and 'agile' principles with a first focus on cost-savings. This is a short-sighted implementation plan that does not account for the long-term effects on patients of losing agency and critical relationships. Lean and agile mechanisms for 'processing' patients have neglected to respect patients' privacy and have risked patient safety as healthcare workers scramble to make their quota of rooming patients in a lean healthcare environment. Future plans of healthcare providers to incorporate remote 'video medicine' as further cost savings, have the possibility to impact patient care as doctors may miss a critical diagnosis due to the loss of resolution in a small video window. Furthermore, the condition of 'denial' can impact the accuracy of patients self-reporting their states.

When IoT is applied to health care the outcome will be multiple partially-automated IoT device-based systems, from different vendors that vary from installation to installation. These systems will depend on other systems (and people) for oversight, information and activities required for proper operation in any specific context. These systems will have to work together without being designed specifically to do so. In terms corresponding to the four principles of Thing Theory (Table 1), we will need a supervisor agent that operates over the entire context. This agent will monitor dynamically the capabilities and requirements of each IoT device system in the context. It will identify appropriate applications of these capabilities as specific and unique circumstance transpire, coordinating all the systems in that context through relevant information and judgements in the context of use.

Thing is, however, just a critical element, not the only element needed for successful IoT applications in healthcare. In short, automation without context, agency and consideration for patients will lead to unforeseen complications that will cost much more in the long run to resolve. Technology that inhibits a physician from developing the trusted relationships critical and foundational to good care, can also create similar problems. The people designing and implementing healthcare systems must see the people within the systems (workers, physicians, and patients) as a series of long-term relationships that are to be managed, not necessarily resources, services and supplies to be coordinated in the moment. Much of what makes a good doctor-patient relationship function well is the understanding and shared history that happens between the doctor and patient over time. IoT technologies can augment relationships by being smarter about how information may be gathered and processed, but it is best to do this within the context surrounding existing and well-established relationships within the healthcare system. Humans do not necessarily benefit from solely interacting with machines in this 
context. However, machines can provide processing and knowledge management that assists and bolsters relationships. Our recommendation is that clinics understand how to augment their offerings to let machines do what they do best in the context of enabling the people within the system to do what they do best: create, build and sustain human relationships in the context of maintaining overall health and well-being.

\section{REFERENCES}

Ahsan, K., Kingston, P., \& Shah, H. (2009). Context Based Knowledge Management in Healthcare: An EA Approached. In AMCIS 2009 Proceedings (pp. 297-302). AIS Electronic Library.

Applin, S. A., and Fischer, M.D. (2011a). A Cultural Perspective on Mixed, Dual and Blended Reality. In Proceedings of the 16th International Conference on Intelligent User Interfaces (IUI'11) IUI

Workshop on Location Awareness for Mixed and Dual Reality (LAMDa'11) (pp. 1-4). Palo Alto, CA: DFKI.

Applin, S. A., and Fischer, M.D. (2011b). Pervasive Computing in Time and Space: The Culture and Context of 'Place' Integration. In Proceedings of the 2011 7th International Conference on Intelligent Environments (IE’11) (pp. 285-293). Nottingham, UK: IEEE.

Applin, S. A., and Fischer, M.D. (2012a). PolySocial Reality: Prospects for Extending User Capabilities Beyond Mixed, Dual and Blended Reality. In Proceedings of the 17th International Conference on Intelligent User Interfaces (IUI'12) Workshop on Location Awareness for Mixed and Dual Reality (LAMDa'12) (pp.1-4). Lisbon, Portugal: DFKI.

Applin, S.A. and Fischer, M.D. (2012b). Applied Agency: Resolving Multiplexed Communication in Automobiles. In Adjunct Proceedings of the 4th Annual Conference on Automotive User Interaction (AutomotiveUI '12) (pp. 159-163), Portsmouth, N.H: ACM SIGCHI.

Applin, S.A. and Fischer, M.D. (2013). Thing Theory: Connecting People to Location-Aware Smart Environments. In Proceedings of the 18th International Conference on Intelligent User Interfaces (IUI'13) Workshop on Location Awareness for Mixed and Dual Reality (LAMDA'13) (pp.115-118). Santa Monica, CA: ACM.

Atzori, L., Iera, A., \& Morabito, G. (2011). Siot: Giving a social structure to the internet of things. Communications Letters, IEEE, 15(11), 1193-1195.

Babar, S., Mahalle, P., Stango, A., Prasad, N., \& Prasad, R. (2010). Proposed security model and threat taxonomy for the internet of things (IoT). In Recent Trends in Network Security and Applications (pp. 420-429). Berlin/Heidelberg: Springer.

Bradshaw, J. M., Hoffman, R. R., Woods, D. D. \& Johnson, M. (2013). The Seven Deadly Myths of "Autonomous Systems". In IEEE Intelligent Systems (54-61). IEEE.

Castro, P. and T. Maibaum. (2007). A complete and compact propositional deontic logic. In Theoretical Aspects of Computing-ICTAC 2007 (109-123). Berlin/Heidelberg, Germany: Springer.

Chaudhry, B., Wang, J., Wu, S., Maglione, M., Mojica, W., Roth, E., Morton, S.C. \& Shekelle, P. G. (2006). Systematic review: impact of health information technology on quality, efficiency, and costs of medical care. Annals of internal medicine, 144(10), 742-752.

Dix, J. and Fisher, M. (2011).Where logic and agents meet. Annals of Mathematics and Artificial Intelligence 61(1), 15-28.

Dong, H., \& Li, X. (2013). A deontic action logic for complex actions. In Logic, Rationality, and Interaction (pp. 311-315). Berlin/Heidelberg: Springer.

Fairbanks, R. J.,Wears, R. L., Woods, D. D., Hollnagel, E., Plsek, P. \& Cook, R. I. (2014 ). Resilience and resilience engineering in health care. Jt Comm J Qual Patient Saf, 40, 376-83.

Fischer, M. D. (2006). Cultural agents: a community of minds. In Engineering Societies in the Agents World VI (pp. 259-274). Berlin/Heidelberg: Springer.

Gubbi, J., Buyya, R., Marusic, S., \& Palaniswami, M. (2013). Internet of Things (IoT): A vision, architectural elements, and future directions. Future Generation Computer Systems, 29(7), 1645-1660. 
Hare, B., \& Tomasello, M. (2005). Human-like social skills in dogs? Trends in cognitive sciences, 9(9), 439-444.

Horty, J. (2001). Agency and Deontic Logic. Oxford: Oxford University Press.

IBM Software (2015). Smarter Process: Reinventing Business Operations. Retrieved from https:// www.youtube.com/watch?v=nZjIApCIUKw (57:57).

Mori, M. (2012/1970).The Uncanny Valley. Trans. Karl F. McDorman and Norri Kageki. IEEE Robotics and Automation Magazine, 19.

Perry, S. J., Mosher, H. J., Persoon, T. J., Bass, E. J., Fairbanks, R. T. J. \& Pennathur, P. R. (2013). Healthcare Systems Design At a Crossroads: Challenges, Opportunities and Strategies. In Proceedings of the Human Factors and Ergonomics Society Annual Meeting (57: pp. 713-717), SAGE Publications

Ryan, M., Fiadeiro, J., \& Maibaum, T. (1991). Sharing actions and attributes in modal action logic. In Theoretical Aspects of Computer Software (pp. 569-593). Berlin/Heidelberg: Springer.

Wears, R. L., \& Leveson, N. G. (2008). Safeware": safety-critical computing and healthcare information technology. Advances in patient safety: new directions and alternative approaches, 4, 110.

\section{KEY TERMS AND DEFINITIONS}

Asynchronous: Not occurring in the current time. Voice mail is an example of an asynchronous message.

Heterogeneity/Heterogeneous: Composed of many different pieces, parts or attributes.

Location-aware: A condition by which technology can track its location in an automated fashion using a combination of hardware, sensors, and software.

Meta-agent: A software algorithm that can monitor various inputs from various lower level hardware sensors and their data outputs.

Multiplexed: A telecommunications term referring to a circumstance where multiple signals, message streams or data, are combined into one signal that is transmitted over a shared medium.

PolySocial Reality: A conceptual model of all synchronous and asynchronous communications by humans/humans, humans/machines, machines/machines, animals/animals, animals/humans, animals/ machines.

Smart Environment: An environment that contains responsive electronic monitor equipment such as sensors, actuators or other devices that can measure information in the environment and act upon it to either generate, share or transmit data to enable reactions from the environment or devices related to the environment.

Synchronous: Occurring in real time in the present. People meeting face-to-face are doing so in synchronous time.

Thing-agent: A trusted meta-agent that manages low level processes in a smart environment and brokers preferences to other trusted meta-agents.

\section{ENDNOTES}

This chapter is derived from an Intelligent User Interfaces (IUI 2013) workshop paper on Location Awareness for Mixed and Dual Reality (LAMDa '13), which the authors have extended for the healthcare smart environment context. The authors thank the Economic and Social Research council for funding for underlying research under grant ES/J012300/1 and the Natural Environment Research Council for funding underlying research under grant NE/ I004149/1. 